\title{
Angry Apps: The Impact of Network Timer Selection on Power Consumption, Signalling Load, and Web QoE
}

\author{
Christian Schwartz, Tobias Hoßfeld, Frank Lehrieder, and Phuoc Tran-Gia \\ Institute of Computer Science, University of Würzburg, Chair of Communication Networks, Am Hubland, \\ 97074 Würzburg, Germany
}

Correspondence should be addressed to Tobias Hoßfeld; hossfeld@informatik.uni-wuerzburg.de

Received 2 November 2012; Revised 23 January 2013; Accepted 13 February 2013

Academic Editor: Pedro Merino

Copyright (C) 2013 Christian Schwartz et al. This is an open access article distributed under the Creative Commons Attribution License, which permits unrestricted use, distribution, and reproduction in any medium, provided the original work is properly cited.

\begin{abstract}
The popularity of smartphones and mobile applications has experienced a considerable growth during the recent years, and this growth is expected to continue in the future. Since smartphones have only very limited energy resources, battery efficiency is one of the determining factors for a good user experience. Therefore, some smartphones tear down connections to the mobile network soon after a completed data transmission to reduce the power consumption of their transmission unit. However, frequent connection reestablishments caused by apps which send or receive small amounts of data often lead to a heavy signalling load within the mobile network. One of the major contributions of this paper is the investigation of the resulting tradeoff between energy consumption at the smartphone and the generated signalling traffic in the mobile network. We explain that this tradeoff can be controlled by the connection release timeout and study the impact of this parameter for a number of popular apps that cover a wide range of traffic characteristics in terms of bandwidth requirements and resulting signalling traffic. Finally, we study the impact of the timer settings on Quality of Experience (QoE) for web traffic. This is an important aspect since connection establishments not only lead to signalling traffic but also increase the load time of web pages.
\end{abstract}

\section{Introduction}

Together with the wide-spread usage of smartphones in today's UMTS networks, the popularity of smartphone apps has seen a tremendous growth during the last years [1]. The resulting traffic is expected to exceed half of the global mobile data traffic in the next years [2]. One of the major reasons for this phenomenon is that smartphones are very convenient for users to stay always connected to the Internet. In turn, this has led developers of smartphone apps to the assumption of continuous Internet connectivity. Therefore, many apps such as social network clients, weather forecasts, or instant messengers update their status frequently, which raises a number of problems-in the mobile network as well as on the smartphones.

In contrast to desktops or laptops, smartphones are equipped only with limited battery. Since established connections from the smartphone to the mobile network consume a large amount of energy, some smartphones close these connections soon after the data transmission is finished, that is, after a very short period of no traffic activity, which is controlled by an inactivity timer. This saves energy and prevents battery drain caused by established but unused connections. However, it might also degrade the user experience since the connection start-up delay is in the order of a few seconds. Therefore, users might get annoyed, for example, if the load time of every web page in their browser is increased. Hence, two oppositional effects impact the quality of experience (QoE) perceived by the user: lower energy consumption and short page load times-a tradeoff that we investigate thoroughly in this paper.

From a mobile operator's point of view, each change between connected and disconnected states of the smartphone causes signalling in the network, and excessive signalling load has led to severe problems in the recent past. For example, a large mobile network in Japan suffered from an outage of several hours in January 2012. The operator of this network mentioned a large number of small control 
messages of certain popular apps (such as keep-alive messages or buddy list updates in VoIP apps) as a probable cause for this outage (Penn-Olson, "Finding a connection: Android, Line, and Docomo's network outage," 2012, available at http://www.penn-olson.com/2012/01/30/docomo-outageline/). Therefore, we argue that not an overload in data traffic but the high number of establishments and teardowns of wireless connections has brought down the network. As a consequence, the network operator may adjust the timer setting accordingly. A longer timer setting can reduce signalling load and resource costs of the mobile operator, however, at the cost of energy consumption of the user device and an increased consumption of radio resources. Hence, an additional tradeoff exists between battery efficiency on smartphones and signalling load in the mobile network. The proprietary fast dormancy mode as implemented by smartphones (i.e., smartphones tear down the connection earlier than advised by the network) additionally affects the signalling load in the network and is also investigated in this paper. The influence of traffic generated by applications on radio resources is another point of interest of mobile operators which is already well investigated in the literature, for example [5], and out of scope in this paper. In light of current troubles with network outages induced by signalling storms, the interest of mobile operators and equipment vendors has shifted towards signalling generated by the application traffic $[6,7]$ on which we focus here.

A reason for this signalling storm is the following. UMTS networks are designed to provide wireless, high bandwidth Internet access for mobile users, for example, video telephony. Therefore, high load in terms of data traffic was carefully considered during the design of such networks. However, some currently popular apps such as Aupeo radio streaming or Skype VoIP apps load the mobile network all the time, even if they are only running in the background. They send update and keep-alive messages every few seconds, which is problematic for today's UMTS networks. The reason is that the mobile network usually tears down wireless connections to a user equipment (UE) after an inactivity timeout of a few seconds, that is, when no data was transmitted for this short time. If the time between two such keep-alive messages is slightly above the connection timeout of the mobile network, the wireless connections between the UE and the mobile network are established and torn down every few seconds. This is an issue that UMTS networks have not been designed for.

The contributions of this paper are the following. First, we study the tradeoff between energy consumption at the smartphone and the generated signalling traffic in the mobile network. From the user's point of view, battery efficiency is one of the determining factors for a good QoE due to a very limited energy capacity of smartphones [8]. Thereby, we analyse the impact of the inactivity timer as well as of the fast dormancy mode for exemplary smartphone apps based on measurements in a public $3 \mathrm{G}$ network. These apps are selected to cover a wide range of traffic characteristics in terms of bandwidth requirements and resulting signalling traffic. In particular, we consider background applications (like Twitter or Skype in passive mode), bandwidth intensive (like Aupeo radio streaming), and interactive applications (like Angry Birds). Then, we answer the question whether a mobile operator is able to find optimal values for the inactivity timer for a given application or network configuration. Further, we see which apps (and which traffic characteristics) make the smartphone users and the mobile operators angry, respectively. We compare the results from these different applications and study the impact of choosing one such optimum for one specific application on other applications. This comparison sheds new light on the practice of using network parameters to optimise for power consumption or generated amount of signalling frequency. Furthermore, we discuss new paradigms for the design of mechanisms which try to resolve such conflicts, namely, economic traffic management [9] and design for tussle [10, 11]. Finally, we study the impact of the timer settings on QoE for web browsing based on existing models for web browsing [12]. This is an important aspect since connection establishments do not only lead to signalling traffic but also increase the load time of web pages which is the key influence factor on web QoE.

The remainder of this paper is structured as follows. Section 2 provides the background on UMTS networks and the radio resource control protocol used for connection establishment and teardown. Related work on measurement studies of relevant RRC parameters like the inactivity timer is reviewed. Further, existing optimisation approaches of the resource consumption are revisited. Then, energy consumption as key QoE influence factor of smartphone users is considered. Section 3 describes the measurement setup and the algorithm to infer (connectivity and energy) state transitions of the smartphone from measured IP packets. Then, we calculate signalling frequency and power consumption based on the state transitions. Section 4 presents the numerical results of our analysis. The traffic of the four popular smartphone apps are characterized. Afterwards, we compare the signalling frequency and power consumption depending on the network configuration. Finally, we study the influence of network parameters on QoE for web browsing based on page load times. Section 5 concludes this work with an outlook on open challenges of how to optimise mobile networks without annoying users or operators.

\section{Background and Related Work}

This section provides background information on the structure of UMTS networks and their components. In addition, it explains the radio resource control (RRC) protocol, which is used for the allocation of wireless transmission resources. Afterwards, it reviews related work on measurement studies of RRC parameters in real networks and on optimisation approaches of such resources, both in the energy and the wireless domain. Finally, we discuss the impact of energy consumption on mobile devices such as smartphones on the QoE of the end user.

2.1. UMTS Networks and the RRC Protocol. UMTS networks consist of the UEs, the radio access network (RAN), and the core network $(\mathrm{CN})$. Their basic structure for packet-switched 


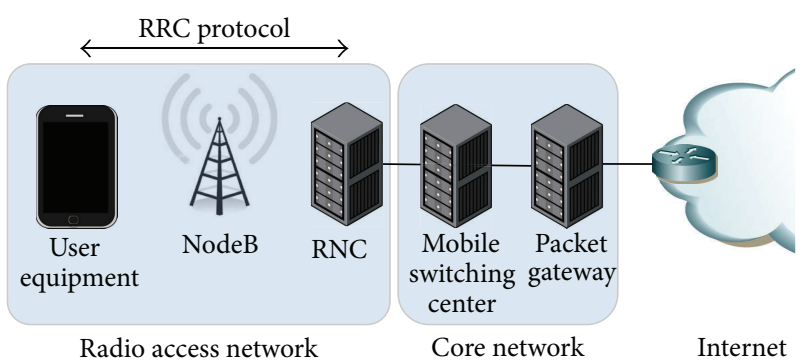

FIGURE 1: Basic structure of a UMTS network for data calls.
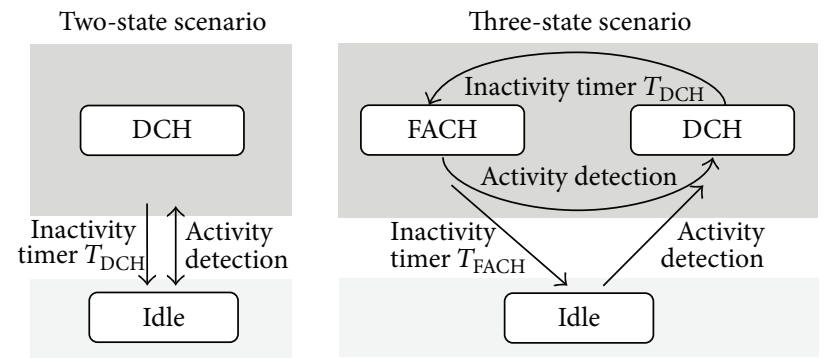

FIGURE 2: RRC state transition diagrams for the two-state and threestate models.

connections is illustrated in Figure 1. UEs are usually cell phones or computers connected using datacards. The RAN connects the UEs to the CN, which is connected to the Internet. The RAN contains the NodeBs in which the UEs are connected to using the air interface. The NodeBs in turn are connected to radio network controllers (RNCs), which among other tasks are responsible for radio resource control using the RRC protocol [3].

For resource management reasons, a UE may be in one of several RRC states, where each state allows the UE a different level of connectivity to the RAN. The RRC protocol specifies five states for the connection between the UE and the RAN: Idle, URA-PCH, CELL-PCH forward access channel $(\mathrm{FACH})$, and dedicated transport channel (DCH).

In the Idle state, the cell is notified of the UE presence, but the UE can neither send nor receive data. The FACH and DCH states allow communication with the RAN, where the $\mathrm{DCH}$ state allows for larger bandwidth at the cost of a higher power consumption. For simplicity reasons, we neglect URAPCH and CELL-PCH in this study. While URA-PCH plays only a role in scenarios of high mobility, CELL-PCH is not yet widely implemented. Our results are still of general nature and do not depend on the limited number of considered RRC states.

State transitions are controlled by the RNC using rules specified in the RRC protocol and timer values set by the network operator [5, 13]. A brief description is given in the following. We consider two different state transition models, depicted in Figure 2. The first model includes the Idle, $\mathrm{FACH}$, and DCH states (cf. the right part of the figure). Therefore, we call it a three-state model. If the UE is in the Idle state and activity is detected (i.e., a packet is sent or received), the connection transits to the $\mathrm{DCH}$ state. After each transmission, a timer $T_{\mathrm{DCH}}$ is started, and it is reset whenever a new packet is sent or received. If the timer expires, the connection transits to the FACH state. Upon entering, the $T_{\mathrm{FACH}}$ timer is started. If a new transmission occurs, the connection again transits to the DCH state. If $T_{\mathrm{FACH}}$ expires, the connection transits to the Idle state.

The second model, denoted as the two-state model, only includes the Idle and DCH states. If the UE is in the Idle mode and a packet is sent or received, the connection transits to the $\mathrm{DCH}$ state. Once in the $\mathrm{DCH}$ mode, the $T_{\mathrm{DCH}}$ timer is started, and it is reset whenever a new packet is sent or received. If the timer expires, the UE transits back to the Idle state.

While the three-state model is closer to the specified RRC protocol, the two-state model is similar to some proprietary fast dormancy implementations used by UE vendors. In these fast dormancy implementations, the UE tears down the connection to the network state as soon as no data is ready to be sent for a certain time, that is, it forces the network to transit to the Idle state. In contrast to the threestate model, there is no transition to the $\mathrm{FACH}$ state. If a device disconnects from the network by transitioning to the Idle state, it has to be reauthenticated before another transition to the DCH state occurs. This results in additional signalling traffic and causes more load on the network [6] due to frequent reestablishments of the RRC connection. These proprietary fast dormancy algorithms do not adhere to the RRC specification [14] but, nonetheless, exist in the real world and have been identified as possible causes for signalling storms. The major reason for fast dormancy implementations is the decrease in power consumption on the UE, since the transmission unit of the UE consumes only $1 \%-2 \%$ of the energy in the Idle state compared to the $\mathrm{DCH}$ state. Thus, both models warrant further investigation.

\subsection{Related Work on Measurements of RRC Parameters and} Optimisation of Resource Consumption. The increased use of mobile broadband networks has caused radio resource management to become a hot research topic. In particular, a considerable research effort is spent on measurements of RRC configurations observed in real UMTS networks. The authors of [13] present a measurement tool exactly for that purpose. They use round-trip times of data packets to infer the RRC state of the connection. This is possible since the latency in the $\mathrm{FACH}$ is significantly higher than in the $\mathrm{DCH}$ state. This allows the authors to measure RRC state transition parameters such as $T_{\mathrm{DCH}}$ and $T_{\mathrm{FACH}}$, channel setup delays, and paging delays in different networks. They find that the settings vary by network and give values of 1.2 seconds for the $\mathrm{DCH}$ release timer and values of more than one minute for the Idle timer. To validate their method of inferring the RRC states from the round-trip times, they compare the actual energy consumption of the device with the expected energy consumption in a specific RRC.

A similar approach is applied by the authors of [5]. They reach comparable conclusions but report timer values of 5 seconds for $T_{\mathrm{DCH}}$ and 12 seconds for $T_{\mathrm{FACH}}$. Furthermore, the authors identify sets of RRC state transitions from two network providers. We used these results to define the state models in Section 2.1. 
In [15], the authors propose the tail optimisation protocol. The main idea is that the applications on the UE can accurately predict whether traffic will soon be transmitted or not. This knowledge permits to avoid the unused tail of $\mathrm{DCH}$ periods if no further data has to be sent. If traffic activity is expected within a short time frame, the UE stays in the DCH state to avoid frequent connection reestablishments and the associated signalling load. The same authors extend this idea in [4] and propose $\mathrm{ARO}$, an application resource optimiser, together with an implementation for Android 2.2. This tool also includes additional features such as batching up data or increasing the update rate of application to optimise their resource consumptions.

Finally, the 3GPP has released a technical report [16] about the adverse impact of mobile data applications. This report states that frequent connection reestablishments due to small data packets caused, for example, by status updates of social network or instant messaging apps, can lead to problems of increased signalling load. This highlights the importance of this topic.

2.3. Smartphone Energy Consumption and Quality of Experience. In [8], the authors performed a 4-week long study with 29 participants to identify factors influencing QoE of mobile applications. The study comprises (1) data from context sensing software, (2) user feedback using an experience sampling method several times per day, and (3) weekly interviews of the participants. To determine the factors of influence, the authors analyse the frequency of specific keywords in the interviews and the surveys. It turns out that the term battery has the highest frequency. According to [8], this is reasonable since the battery efficiency has a strong impact on the user perceived quality, in particular, when it is nearly discharged.

As a consequence of this finding, we investigate the energy consumption of a smartphone as one of the main indicators for QoE. In addition, we show that the energy consumption of the transmission unit of a smartphone can be reduced if the state of wireless connection between the UE and the RNC is set to Idle shortly after data transmission. However, this can lead to frequent connection reestablishments since some apps send or receive small status updates very often. The signalling load produced by such issues is one of the major problem in today's UMTS networks. Hence, this is clearly a tradeoff between battery efficiency on the side of the end user and the network load for the network operators.

There are different approaches to cope with such tradeoffs in the design of specific mechanisms. The two most prominent ones are economic traffic management (ETM) [9] and design for tussle $[10,11]$. The ETM paradigm suggests that the different stakeholders collaborate and exchange information so as to permit a joint optimisations of the tradeoff. This can achieve better results than separate optimisation since these tend to work in opposite directions. Design for tussle focuses more on conflicting interests than on cooperation. It means that mechanisms should be able to adapt the outcome of a certain tussle at run time and not at design time, for example, by giving the sole control of essential entities to a certain stakeholder.

\section{Inferring Signalling Frequency and Power Consumption from Network Trace}

However, RRC state transitions are triggered by the UE's firmware. While solutions exist to capture RRC state transitions on specific hardware [17], they are not available for all modern smartphone platforms. Other options to measure the required information include using costly hardware and specific UEs, usually not available to researchers and developers. This prevents the application developers from evaluating the effect their applications have on the overall health on the network. Consequently, they cannot take measures to prevent the harmful behaviour of their applications. However, it is possible to infer the RRC state transitions for a given packet trace if the network model is known.

In this section, we first describe the setup used to capture network packet traces for arbitrary apps. Then, we give an algorithm to infer the RRC state transitions for a given packet trace. Based on these state transitions, we can calculate the number of signalling messages generated by the packet trace. Finally, we use the information of the RRC state of the UE at every given point in time to calculate the power consumption of the UE's radio interface.

3.1. Measurement Procedure and Setup. To investigate the behaviour of the application under study, we capture traffic during a typical use of the application on a smartphone. The smartphone runs the Android operating system and is connected to the $3 \mathrm{G}$ network of a major German network operator. To obtain the network packet traces, we use the tcpdump application. This application requires root privileges which are obtained by rooting the device and installing the custom cyanogenMod ROM (http://www.cyanogenmod.org/). Once tcpdump is installed and running, we start the application under study and capture packet traces while the application is running. Then, the android debugging bridge is used to copy the traces to a workstation. The traces contain Internet Protocol (IP) packets as well as Linux Cooked Captures. We only require the IP packets; thus, we filtered the traces for IP packets which are used during the following analysis.

3.2. Inferring Network State. In this section, we study the influence of the application traffic on RRC state transitions and signalling messages. Since RRC state transitions cannot be captured using commonly available tools, we introduce an algorithm to infer RRC state transitions from IP packet traces. Using this algorithm, we analyse the RRC state transition frequency and signalling message load for the two-state model and the three-state model.

Traffic below the network layer cannot be measured without specific equipment which is often out of reach for developers interested in assessing the impact of their applications on the network. Based on the two-state and the three-state models introduced in Section 2.1, we process tcpdump captures of the application traffic. However, it should be noted that this method is not restricted to a specific network model but can be extended to any other network model as well. Using these captures, we extract the timestamps when IP packets are sent or received. Furthermore, 


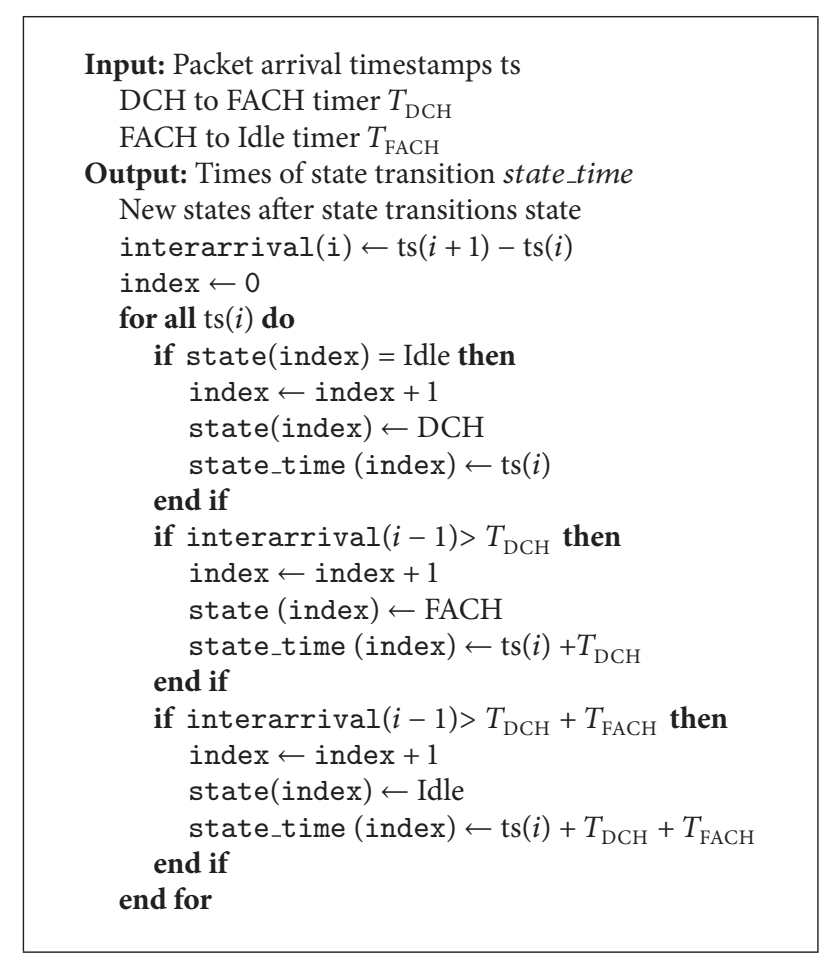

Algorithm 1: Inferring RRC state transitions based on IP timestamps.

we require the timer values of the transition from the $\mathrm{DCH}$ state to the $\mathrm{FACH}$ state, $T_{\mathrm{DCH}}$, and the timer for the transition between the FACH and the Idle states, $T_{\mathrm{FACH}}$. Based on this information, Algorithm 1 infers the timestamps of state transitions according to the 3GPP specification [3] for the three-state model. This algorithm can be simplified to also work for the two-state model. Alternatively, a way to postprocess the results of the algorithm to obtain results for the two-state model is given at the end of this section. The algorithm first computes the interarrival times for all packets. Then, each timestamp is considered. If the UE is currently in the Idle state, a state transition to DCH occurs at the moment the packet is sent or received. If the interarrival time exceeds the $T_{\mathrm{DCH}}$ timer, the UE transits to the FACH, $T_{\mathrm{DCH}}$, seconds after the packet was sent or received. Similarly, if the interarrival time exceeds both the $T_{\mathrm{DCH}}$ and $T_{\mathrm{FACH}}$ timers, a state transition to Idle occurs, $T_{\mathrm{FACH}}$, seconds after the state transits to the FACH.

UE vendors always search for ways to decrease energy consumption of their devices. A straightforward way to achieve this, if only the wellbeing of the UE is considered, is to transit from the DCH to Idle states as soon as no additional data is ready for sending. While this transition is not directly available in the 3GPP specification for the RRC protocol [3], a UE may reset the connection, effectively transitioning from any state to Idle. This behaviour can be modelled using the two-state model introduced in Section 2.1.

State transitions for the two-state model can be calculated using a similar algorithm. Alternatively, the behaviour of the two-state model can be emulated using Algorithm 1 if $T_{\mathrm{FACH}}$
TABLE 1: Number of signalling messages per RRC state transition perceived at the RNC (taken from [3]).

\begin{tabular}{lccc}
\hline From/to & Idle & FACH & DCH \\
\hline Idle & - & 28 & 32 \\
FACH & 22 & - & 6 \\
DCH & 25 & 5 & - \\
\hline
\end{tabular}

TABLE 2: Power consumption of the UE radio interface depending on current RRC state (taken from [4]).

\begin{tabular}{lc}
\hline RRC state & Power consumption $(\mathrm{mW})$ \\
\hline Idle & 0 \\
FACH & 650 \\
DCH & 800 \\
\hline
\end{tabular}

is set to 0 seconds and all state transitions to $\mathrm{FACH}$ are removed in a postprocessing step.

\subsection{Calculating Signalling Frequency and Power Consumption.} In reality, the number of state transitions is not the metric of most importance if network load should be evaluated. Each state transition results in a number of RRC messages between the UE and different network components. For this study, we consider, the number of messages perceived at the RNC, which can be found in [3] and is summarized in Table 1 . It can be seen that transitions from or to the Idle state are especially expensive in terms of number of messages sent or received. This is due to the fact that upon entering or leaving, the Idle state authentication has to be performed. Note that for the two-state model, only transitions from or to the Idle state occur. This results in the fact that for the same network packet trace, the number of signalling messages occurring in the two-state model is generally higher than in the three-state model. To obtain the total number of signalling messages, we weight the number of state transitions with the number of messages sent per state transitions. Then, we average the number of state transitions over the measurement duration to obtain a metric for the signalling load at the RNC. The inference algorithm does not differentiate between state changes caused by upstream or downstream traffic. State changes caused by downstream traffic usually generate some additional signalling messages, as paging is involved. The inference algorithm can be easily enhanced to support this behaviour. However, the results discussed in the next section would only change quantitatively. Furthermore, the inference of signalling messages can be easily adapted to new networking models or signalling numbers.

From users' point of view, the signalling message frequency is not important. The user is interested in a low power consumption because this increases the battery time of the device. To calculate the battery time, we use the time when state transitions occurred, and the new RRC state of the UE to calculate the relative amount of time that was spent in each state. Given the relative time spent in each state, we use Table 2 (taken from [4]) to compute the power consumption of the radio interface during the measurement phase. To obtain the energy consumption, the power consumption can 
TABLE 3: Qualitative characterization of applications under study.

\begin{tabular}{|c|c|c|c|}
\hline Application & $\begin{array}{c}\text { Traffic } \\
\text { characteristic }\end{array}$ & Application use & $\begin{array}{c}\text { Required } \\
\text { bandwidth }\end{array}$ \\
\hline Angry Birds & Interactive & Foreground & $\begin{array}{c}\text { Low } \\
\text { bandwidth }\end{array}$ \\
\hline Aupeo & Interactive & Background & $\begin{array}{c}\text { High } \\
\text { bandwidth }\end{array}$ \\
\hline Twitter & $\begin{array}{l}\text { Periodic, low } \\
\text { frequency }\end{array}$ & Background & $\begin{array}{c}\text { Low } \\
\text { bandwidth }\end{array}$ \\
\hline Skype & $\begin{array}{l}\text { Periodic, high } \\
\text { frequency }\end{array}$ & Background & $\begin{array}{c}\text { Low } \\
\text { bandwidth }\end{array}$ \\
\hline
\end{tabular}

be multiplied with the duration of the measured network packet trace. We only focus on the power consumption of the radio interface, as it is possible to measure the aggregated power consumption using out-of-the-box instrumentation techniques provided by the hardware vendor.

\section{Numerical Results of Measurement Study}

In the measurement study, we apply the methods introduced in Section 3 to four popular smartphone applications to infer signalling traffic and energy consumption. In Section 4.1, we characterize the applications in terms of traffic patterns, application usage, and bandwidth requirements. In Section 4.2, we study the signalling frequency and power consumption caused by these applications, if inactivity timers such as $T_{\mathrm{DCH}}$ or $T_{\mathrm{FACH}}$ are modified. Finally, we analyse in Section 4.3 the influence of network parameters on web QoE in terms of mean opinion score (MOS) depending on page load times which are influenced by the network settings.

\subsection{Characterization of Traffic Patterns for Selected Applica-} tions. For this study, we chose four specific applications in order to cover a broad spectrum of traffic characteristics, as described in Table 3. First, we discuss said characteristics for these applications. We differentiate between applications, where the user interaction causes the generation of traffic, and such where the application periodically sends or receives traffic. Finally, we consider the amount of bandwidth used by the application.

Angry Birds for Android is a popular interactive freeto-play game and runs in the foreground. To finance the game, an advertisement is shown once the player starts or restarts a level. Advertisements are downloaded on demand by the application but require low bandwidth. Thus, the time between two advertisements depends on the frequency of the player advancing to the next level or deciding to restart the current one.

Aupeo is an Internet radio application, allowing a user to listen to content from personalised radio stations while running in the background. Content is not streamed but downloaded at the beginning of the track. The exact duration depends on the radio stations chosen by the user and is thus interactive. This results in large times of inactivity during the playback of the track itself. Due to the fact that audio files are downloaded, there is a high bandwidth requirement.

The Twitter client is used to send and receive new short messages from the user's Twitter account. Transferring these messages requires relatively low bandwidth. To this end, the user can specify an update frequency when to pull new messages in the background. Thus, the downloads occur with a periodic behaviour of low frequency, where the client sends an HTTPS request to the Twitter server and in return receives new Tweets for the user's account. We do not consider an active user who is publishing new Tweets. Such behaviour would manifest as additional traffic to the periodic one generated by the status updates. Due to the fact that publishing updates occurs relatively infrequently and updating the feed occurs more often, the traffic generated by publishing updates is dominated by that occurring due to updates and thus can be neglected.

Finally, we consider the Skype application. We do not consider any Voice over IP (VoIP) calls but the application's Idle behaviour, that is, when the application is running in the background. During this time, the application sends keepalive messages to the network. These keep-alive messages are sent with high frequency and require low bandwidth.

In addition to the applications considered, there exist other categories of applications which are running in the foreground and interactively require a high bandwidth. One example for such an application is Skype while taking a VoIP call. These applications are not considered in this study because this kind of behaviour causes the UE to be always online. This results the minimal amount of signalling messages to be sent and a maximal power consumption at the UE, independent of network model, or used parameters. Other combinations of traffic criteria also exist. However, from both a signalling load as well as a power consumption point of view, they can be mapped to one of the discussed cases. For example, if an application is sending periodic updates with low bandwidth without user interaction, then the fact that the application is running in the foreground or the background is without consequence for the generated signalling load or power consumption. However, these cases should be considered when the optimisation strategies for message sending are under study. For example, background applications could allow for the batching of messages because the transmission is usually not urgent, while foreground applications do not allow for such behaviour because it would decrease QoE.

Next, we describe the applications under study in more detail. For each application, we show the cumulative distribution function (CDF) of the interarrival times in Figure 3(a) and give information about the mean values and standard deviation of both interarrival times and bandwidth in Table 4, respectively.

Let us again begin with the Angry Birds application. We see that there are no distinct peaks in interarrival time, which would hint at periodic behaviour. Furthermore, we see that $5 \%$ of all interarrival times are greater than 1 second. As we consider only $T_{\mathrm{DCH}}$ values above 1 second, those are candidates for triggering state transitions. The mean interarrival time is 0.66 seconds, with a relatively high standard deviation 


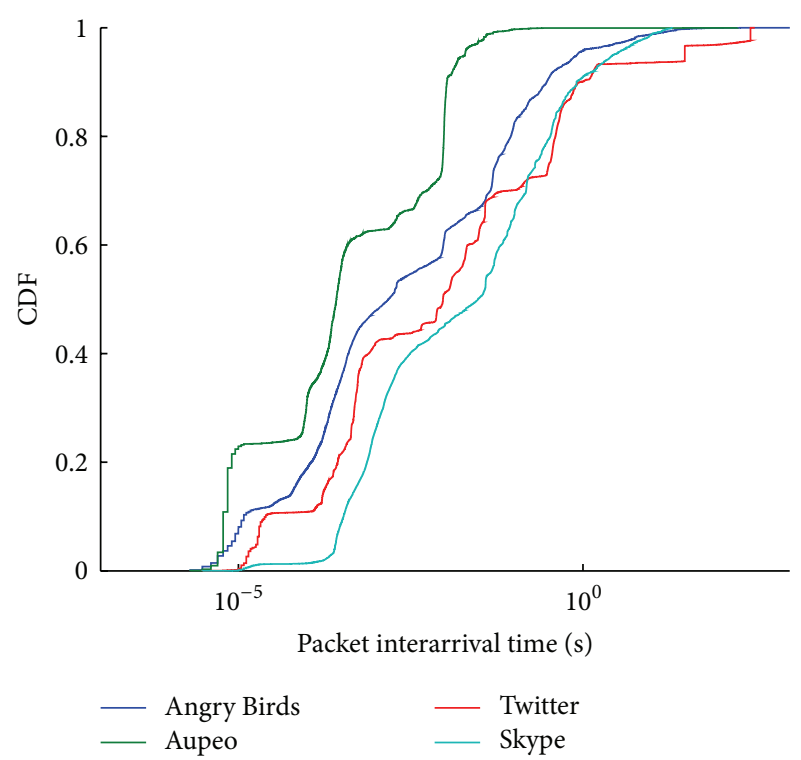

(a) $\mathrm{CDF}$ of interarrival times

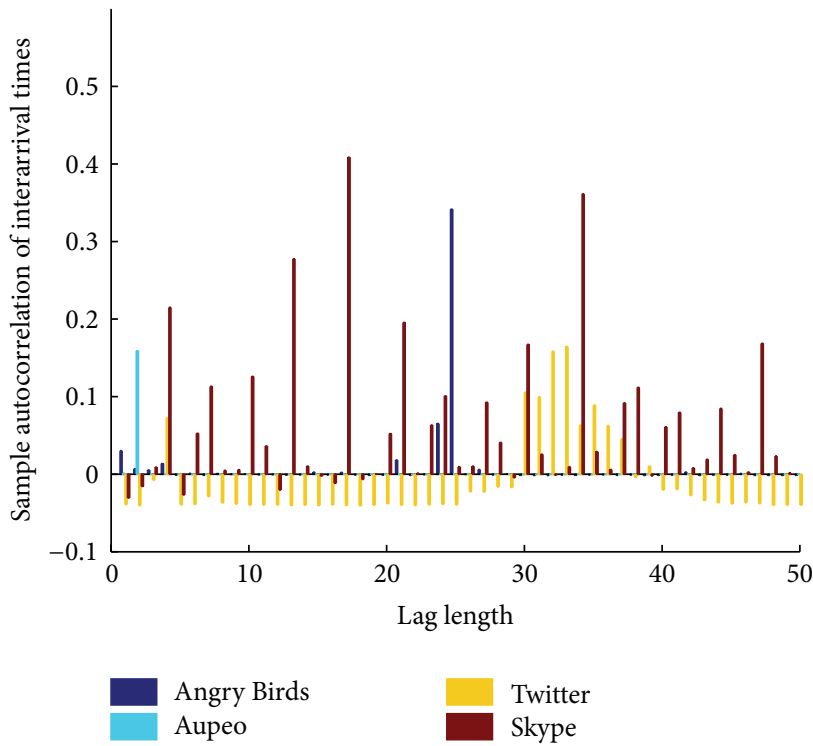

(b) Autocorrelation of interarrival times

FIGURE 3: Characteristics of the four different smartphone applications in our measurement study.

TABLE 4: Mean and standard deviation of interarrival time and bandwidth for considered apps.

\begin{tabular}{lcccc}
\hline Application & $\begin{array}{c}\text { Mean interarrival time } \\
\text { (seconds) }\end{array}$ & $\begin{array}{c}\text { Standard deviation of interarrival } \\
\text { time (seconds) }\end{array}$ & $\begin{array}{c}\text { Mean bandwidth } \\
\text { (kilobit/second) }\end{array}$ & $\begin{array}{c}\text { Standard deviation of } \\
\text { bandwidth (kilobit/second) }\end{array}$ \\
\hline Angry Birds & 0.66 & 15.90 & 4.42 & 4.50 \\
Aupeo & 0.06 & 3.06 & 129.76 & 482.63 \\
Twitter & 8.91 & 44.09 & 0.27 & 0.04 \\
Skype & 0.55 & 1.95 & 1.30 & 1.84 \\
\hline
\end{tabular}

of 15.90 seconds. This is caused by the low interarrival times in one advertisement request and the relatively large interarrival times between two advertisements. Mean bandwidth is relatively low with $4.42 \mathrm{kbps}$ and a high standard deviation of $4.5 \mathrm{kbps}$. These differences can be explained by considering the behaviour of the application. During long phases of use, no traffic is sent, and, after a level is restarted, a new advertisement has to be obtained, causing the transmission of data.

Next, we study the behaviour of the Aupeo application. We see that the application generates packets with relatively small interarrival times. This finding is backed up by the small mean interarrival time of 0.06 seconds. The high standard deviation of 3.06 seconds is caused by the wait between two tracks. Furthermore, we see a high mean bandwidth of $129.76 \mathrm{kbps}$ and a standard deviation of $482.63 \mathrm{kbps}$. This is caused by the difference in traffic activity between times when tracks are either downloaded or not.

For Twitter, we see that $90 \%$ of all transmissions occur with an interarrival time of 1 seconds. Also, we can observe a high mean interarrival time of 8.91 seconds and a high standard deviation of 44.49 seconds. Additionally, the mean bandwidth is low with only $0.27 \mathrm{kbps}$ and a low standard deviation of $0.04 \mathrm{kbps}$ due to the fact that Twitter text messages are only 140 characters in length, and thus only a low volume of traffic needs to be transmitted.

Finally, we consider the Skype application. Similar to the Twitter application, we see that $90 \%$ of all packets occur with an interarrival time of less than 1 second. However, in contrast to Twitter, we see a low mean interarrival time of 0.55 seconds with a standard deviation of 1.95 seconds. Further, we observe a relatively low mean bandwidth of $1.30 \mathrm{kbps}$ and a standard deviation of $1.8 \mathrm{kbps}$.

To further study the traffic patterns of the applications, we study the autocorrelation of the packet interarrival time with regard to the lag length in Figure 3(b). We note that all studied applications present completely different autocorrelations for the interarrival times. This is one of the reasons that the applications under consideration will display different signalling behaviour in the next section.

4.2. Influence of Application Characteristics on Optimisation with Network Timers. This section studies the impact of traffic generated by applications on both the network and the QoE of the user. We consider two metrics. First, we consider the frequency of signalling messages perceived at network components such as an RNC. In light of network outages caused by the so called signalling storms (a large 
number of signalling messages leading to overload at network equipment), it is in the interest of a network operator to reduce the number of signalling messages arriving at the RNC. One possible way to reduce the signalling frequency is to modify network timer values like $T_{\mathrm{DCH}}$ and $T_{\mathrm{FACH}}$. As discussed in Section 2.3, the QoE a user perceives while using his device is influenced by the battery life of the UE. Thus, the second metric considered is the influence of the used network model and associated timer settings on the device's power consumption. As described in Section 3.3, based on a measurement trace for an application, we use Algorithm 1 to infer the state transitions occurring during the use of the application. Then, we calculate the relative time spent in each state and use Table 2 to compute the mean power consumption of the radio interface during the measurement. We study both metrics: first on its one and then aggregated for both network models introduced in Section 2.1. First, we consider the three-state model in Section 4.2.1, which describes the default behaviour in $3 \mathrm{G}$ networks. Then, we describe the influence of the two-state model in Section 4.2.2. Here, we model a network behaviour similar to that if proprietary fast dormancy algorithms are used. These algorithms have been identified as one of the causes of a signalling storm [6]. Finally, we summarize the results and discuss the possible ramifications of using network timer values to reduce the signalling frequency in Section 4.2.3. More numerical results and details can be found in [18].

4.2.1. Three-State Model: Signalling Frequency versus Power Consumption. First, we investigate the signalling frequency generated by the studied applications for the three-state network model. Figure 4(a) shows the signalling frequency with regard to the $T_{\mathrm{DCH}}$ timer. For all studies of the three-state model, the FACH timeout is set to $T_{\mathrm{FACH}}=2 \cdot T_{\mathrm{DCH}}$, a realistic value, as shown in [4]. We see that for $T_{\mathrm{DCH}}$ timers shorter than 6 seconds, the Skype application in Idle mode generates the highest signalling message frequency. The Angry Birds application generates the second highest frequency of signalling messages, followed by the Aupeo application. The Twitter application generates the smallest signalling load. If the $T_{\mathrm{DCH}}$ value is longer than 15 seconds, this order changes. However, in general, the signalling message frequency for higher $T_{\mathrm{DCH}}$ timeouts is lower than for shorter $T_{\mathrm{DCH}}$ timeouts. Now, the Aupeo application has the highest signalling frequency, followed by the Twitter application. The signalling message frequency for the Angry Birds application takes the third place. The application which generated the highest signalling message frequency generates the lowest frequency for higher timeout values. This behaviour can be explained by the fact that the Skype application sends keepalive messages with an interval of less than 20 seconds. If the timer is greater than the interval time of the keep-alive messages, the UE stays always connected and thus generates almost no signalling.

These results show that the traffic patterns of the application have a large influence on the generated signalling load. Signalling is generated for every pause in sending or receiving larger than the configured timeouts. If such pauses occur frequently, this increases the signalling load as shown on the examples of Skype and Angry Birds. Applications with more time between the sending or receiving of data cause less signalling, as shown by Aupeo and Twitter. Furthermore, we can observe that the signalling load can be reduced by increasing the $\mathrm{DCH}$ timeout, with the minimum being reached as $T_{\mathrm{DCH}}$ approaches infinity. From a signalling load perspective, a value of 20 seconds would probably be sufficient; however, if other metrics such as radio resource consumption are considered, 10 seconds would be acceptable for a network operator.

Based on this finding, we see that increasing the $T_{\mathrm{DCH}}$ timer decreases the signalling frequency at the RNC. However, the actual signalling frequency depends on the application running at the UE. From a network operator's point of view, the three-state model should always be preferred to the two-state model because it generates less signalling messages per second, thus decreasing the load at the RNC. This view however does not consider the additional radio resources which are kept in use for a longer time if larger $T_{\mathrm{DCH}}$ values are used. Additionally, it should be noted that the choice of the network model is sometimes outside of the domain of the network operator. Proprietary fast dormancy algorithms, as the considered two-state model, are enabled on the UE by the user.

In Figure 5(a), we consider the power consumption if the network uses the three-state model, that is, if the fast dormancy mode of the UE is disabled. The figure shows the mean power consumption of the device with regard to the $T_{\mathrm{DCH}}$ timeout. Possible values range between $0 \mathrm{~mW}$ if the UE was in Idle state during the whole measurement and $800 \mathrm{~mW}$ if the UE was in DCH state during the complete measurement. We see that the least power over all considered $T_{\mathrm{DCH}}$ values is consumed by the Twitter application. The second least power consumption is required by Aupeo, followed by Angry Birds. Finally, the most power is consumed by Skype. Here, we see that the maximum value of $800 \mathrm{~mW}$ is reached at a $T_{\mathrm{DCH}}$ timeout of 20 seconds. This is because, due to the periodic traffic behaviour of Skype, the device is always in the DCH state. Again, we see that the traffic characteristics of the applications impact the power consumption. Applications with more network activity are forced to stay in more power consuming states for a longer time. We see that for very small network timers, the power consumption is minimal. However, as seen in the last section, small timers increase the signalling load at the RNC. Again, a choice of 10 seconds for the $T_{\mathrm{DCH}}$ timer can be seen as a compromise between signalling load and power consumption.

Finally, we aggregate both metrics in Figure 6(a). The $x$-axis of the figure gives the signalling message frequency. On the $y$-axis, we show the power consumption. Different $T_{\mathrm{DCH}}$ values are shown by different colors as specified by the colorbar. First, we consider Angry Birds. We observe that as the signalling frequency approaches zero, the power consumption rapidly increases, even if only small gains in signalling frequency reduction can be achieved. The Aupeo application presents a completely different picture. Here, we can see multiple almost horizontal lines of markers. If $T_{\mathrm{DCH}}$ is chosen in this range, each increase of $T_{\mathrm{DCH}}$ brings a small 


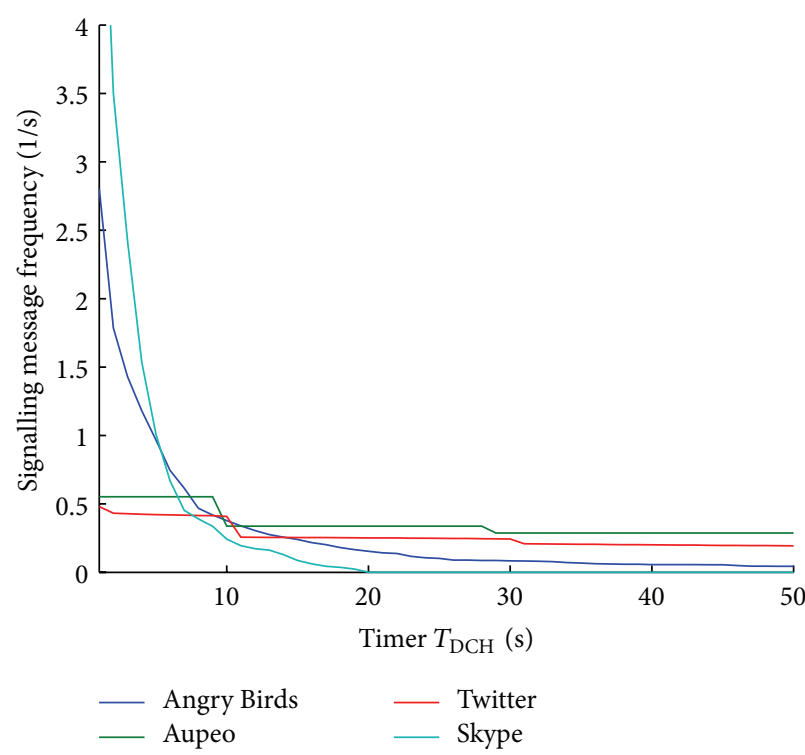

(a) Three-state model

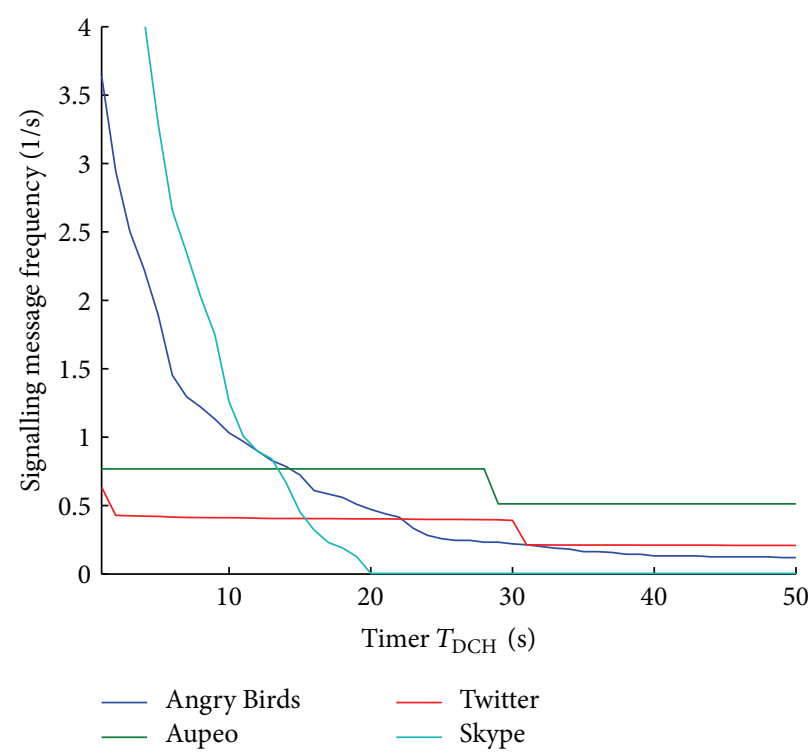

(b) Two-state model

FIGURE 4: Signalling messages frequency for varying $T_{\mathrm{DCH}}$ timers.

decrease in signalling frequency for an increase in power consumption. However, some points of discontinuity exist. If, for example, the DCH timer is increased from 10 seconds to 11 seconds, a decrease in power consumption of $40 \%$ can be achieved by only suffering from a small increase of power consumption. These points of discontinuity would present themselves to be suitable targets of optimisation. Next, we consider the Twitter application. It displays a similar behaviour as the Aupeo application, with multiple points of discontinuity. Note that Twitter exhibits a different point of discontinuity, and the $T_{\mathrm{DCH}}$ value of 10 seconds, which provided good results for Aupeo, is not optimal for Twitter. Finally, Skype shows a completely different picture. First, note that due to the large signalling frequency of Skype for small values of $T_{\mathrm{DCH}}, T_{\mathrm{DCH}}=1$ second is not displayed in the figure. Furthermore, as the $T_{\mathrm{DCH}}$ timer increases above 20 seconds, the signalling frequency does not decrease any further, and the power consumption remains at the maximum value. We observe that there is no common optimal value for all applications which would result in an acceptable tradoff.

\subsubsection{Two-State Model: Signalling Frequency versus Power} Consumption. Now, we study the consequences of the application traffic in a network using the two-state model. The two-state model occurs in reality if fast dormancy implementations are considered. Here, the UE disconnects from the network if for a certain time no traffic is sent or received in order to reduce power consumption. As for the threestate model, Figure 4(b) shows the signalling frequency with regard to the setting of the $T_{\mathrm{DCH}}$ timer. We see the same general behaviour as with the three-state model; however, the signalling frequency generated by each of the applications for the two-state model is usually higher. For example, even for relatively high $T_{\mathrm{DCH}}$ timeout values of 10 seconds, the Angry Birds application causes $270 \%$ of the signalling frequency as in a network using the three-state model.

Next, we consider the changes in the power consumption of the UE if the user decides to enable fast dormancy, that is, switch to a two-state model, in Figure 5(b). As with the signalling frequency, we only see a quantitative differences to the three-state model. Again, we compare the differences between the two-state model and the three-state model on the example of the Angry Birds application. For the same considered $T_{\mathrm{DCH}}$ timeout of 10 seconds, we see a decrease of $81 \%$ in power consumption when compared with the 3 -state model.

Finally, we compare the influence of changes of the $T_{\mathrm{DCH}}$ timeout on both signalling frequency and power consumption for the two-state model in Figure 6(b). As for the three-state model, we see that there is no tradeoff between power consumption and signalling frequency that would be acceptable for all application. Even for single applications, $T_{\mathrm{DCH}}$ values such as 11 seconds which was an acceptable tradeoff for Angry Birds is no longer a good choice in the twostate model.

4.2.3. Consequences of Tradeoff: Signalling Frequency versus Power Consumption. To illustrate the ramifications of the behaviour discussed in the previous section, we compare the influence of the $T_{\mathrm{DCH}}$ timer on an application with different traffic characteristics, for example, the Aupeo application as shown in Figure 7(b). The signalling load before the increase of the DCH timer was 0.55 messages per second; after the change to $T_{\mathrm{DCH}}=8$ seconds, the load remains unchanged. Thus, the policy change based on one application brings no significant gain to other applications. However, from a user's point of view, the power consumption increased 


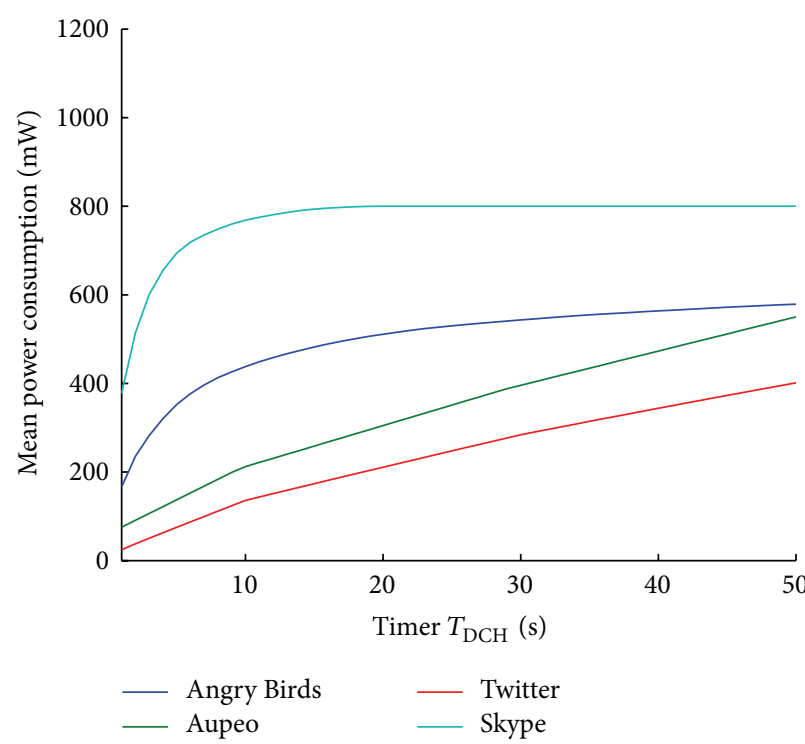

(a) Three-state model

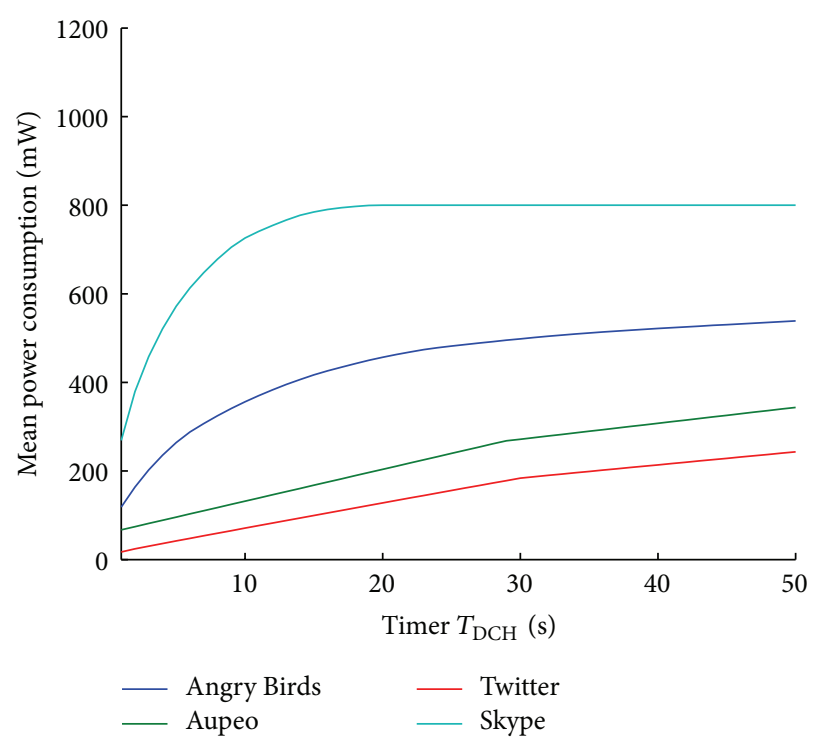

(b) Two-state model

FiguRE 5: Power drain for varying $T_{\mathrm{DCH}}$ timers.

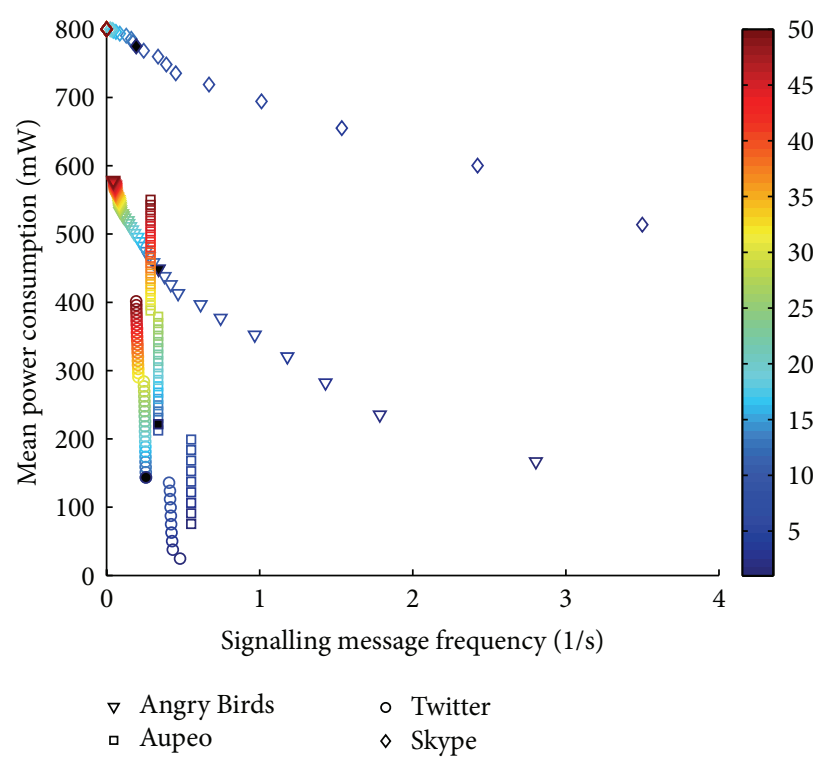

(a) Three-state network model

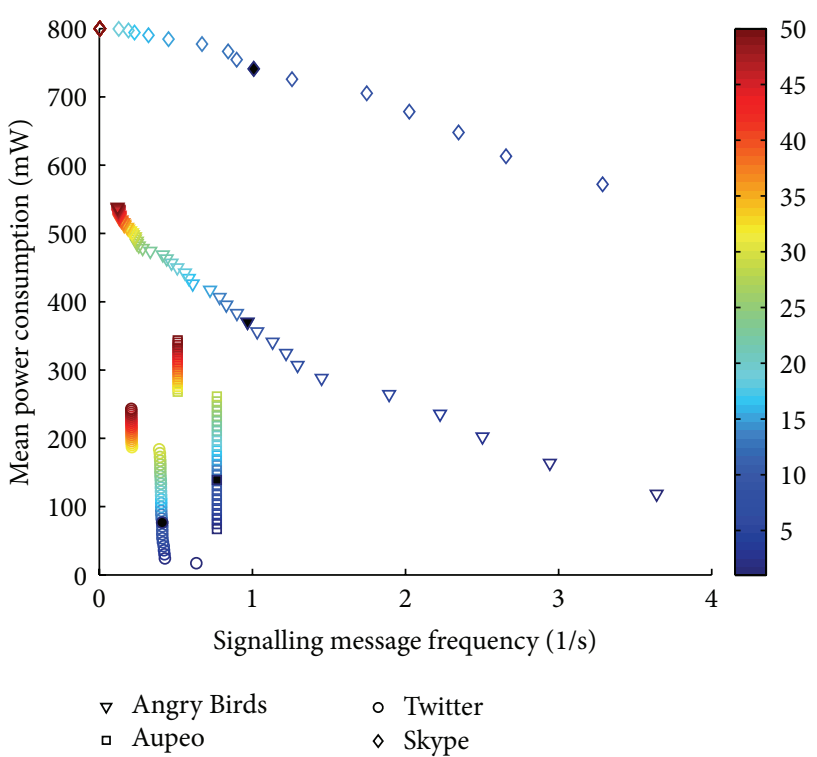

(b) Two-state network model

FIGURE 6: Influence of manipulating $T_{\mathrm{DCH}}$ timer on signalling message frequency and power consumption. Filled marker highlights $T_{\mathrm{DCH}}=$ 11 seconds.

from $121 \mathrm{~mW}$ to $183 \mathrm{~mW}$. Again, we assume that the user activates fast dormancy to deal with the increase in power consumption of more than $50 \%$. This results in a decrease of power consumption to $117 \mathrm{~mW}$ and an increase of overall signalling frequency to 0.76 signalling messages per second. By changing the value without considering all applications, the network operator has decreased the QoE for other users and worsened their overall situation. Thus, due to the large number of applications, it seems impossible to optimise the $\mathrm{DCH}$ timeout to reduce the signalling message frequency without negatively impacting the users QoE in unexpected ways.

There exist applications, like Twitter and Aupeo, where optimisation by modifying the $T_{\mathrm{DCH}}$ values can provide acceptable results. However, these optimisations are only successful if a single application or network model is considered. For other applications, like Angry Birds or Skype, this optimisation approach does not seem to be successful. A reduction of signalling load and power consumption is possible, if the application developers are incentivised to 


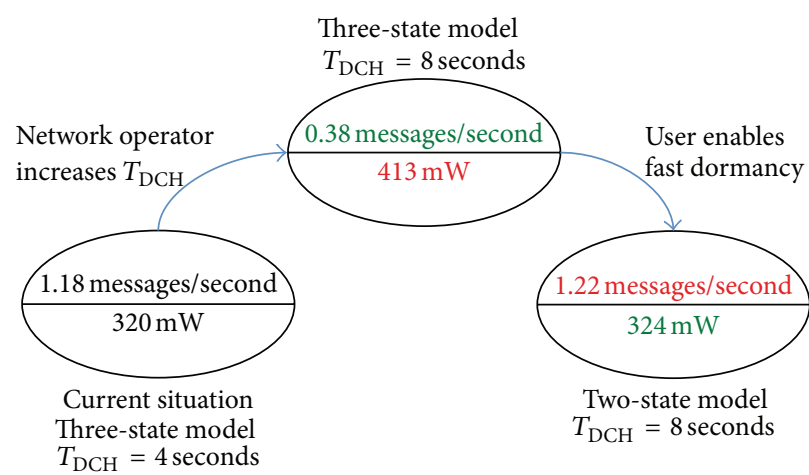

(a) Results of the optimisation on target application Angry Birds

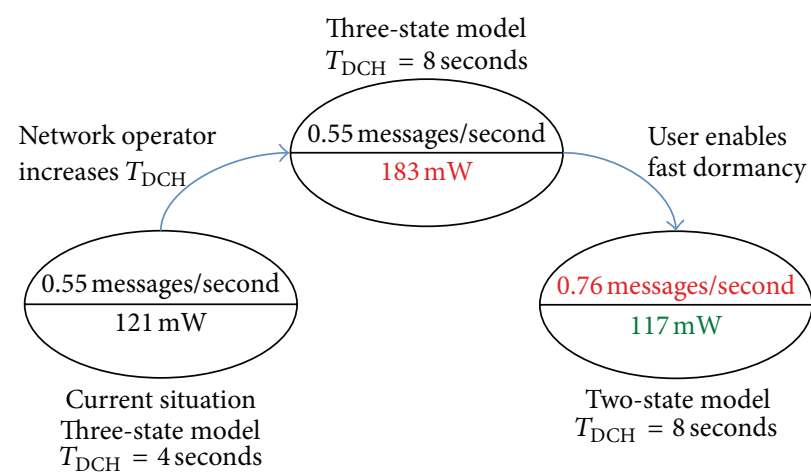

(b) Influence of the optimisation for Angry Birds on Aupeo

FIGURE 7: Influence of manipulating $T_{\mathrm{DCH}}$ timer on different applications.

optimise their applications in these regards. In [4], the authors suggest methods to achieve this optimisation, for example, batch transfer of advertisements for applications like Angry Birds or decreasing the refresh rate in applications like Skype. However, at the moment, neither application developers are receiving incentives to optimise applications in this way, nor hardware vendors do provide interfaces to facilitate such optimisation. Such interfaces would allow application developers to schedule their data transmissions in such a way that both signalling and battery drain would be reduced. Additionally, these interfaces would need to allow the application developer to specify if sending the transmission is urgent because the application is being actively used by the user and requires the feedback of the transmission or if the data is being sent as a regular update, while the application is running in the background and can be scheduled for later transmission as suggested by $[19,20]$.

To bring network operators, hardware vendors, and application developers together and allow for global optimisation of all relevant metrics, holistic approaches like Economic Traffic Management or Design for Tussle, as described in Section 2.3, are required.

\subsection{Impact of Network Configuration and Background Traffic} on Web QoE. So far, we have discussed only power consumption as a QoE influence factor. For applications like web browsing, one relevant QoE influence factor is page load times. Therefore, we consider a web QoE model which quantifies the impact of page load times on mean opinion scores [12]. We distinguish here between web QoE and QoE as no QoE models are currently existing which consider page load times as well as power consumption. In this section, we study the impact of background traffic as well as network timer settings on the page load time of an image and the resulting MOS. For this study, we only consider the threestate network model, but the results can be applied to the twostate model as well.

We assume a scenario where a user is running a background application like Twitter or Skype. Then, while the application is in the background, the user begins to download an image from a website. Due to the background traffic, and depending on the network model and associated timer values, the UE may be currently either in Idle, FACH, or DCH state. We give the probability of a random observer encountering the system in FACH state by $p_{\mathrm{FACH}}$ and the probability of a random observer encountering in Idle state by $p_{\text {Idle }}$. If the device is currently not in DCH state, it takes some time to connect. This promotion time depends on the current state and is according to [15] 2 seconds if the UE is in Idle state and 1.5 seconds if the device is in the FACH state. For this study, we assume that the user randomly chooses a time to begin downloading an image. The time until the image is displayed consists of the time to load the page $t_{p}$ as well as the time to go online $t_{o}$, where $t_{o}$ is the mean time to go online, given as

$$
t_{o}=p_{\text {Idle }} \cdot 2.5 \mathrm{~s}+p_{\mathrm{FACH}} \cdot 1.5 \mathrm{~s} \text {. }
$$

Thus, the total time $t$ that is required to download the image is given by $t=t_{o}+t_{p}$.

The authors of [12] give a function to calculate the MOS based on the required page load time as $\mathrm{QoE}(t)=a$. $\ln t+b$, where $a$ and $b$ depend on the type of content being downloaded. For our scenario, picture download and values of $a=-0.8$ and $b=3.77$ are suggested. It has to be noted that for different websites, the logarithmic function was still observed, but different values for $a$ and $b$ were obtained as given in [12]. These values depend for example on the type of web page as well as the size of the content. Nevertheless, the results presented in this section are therefore generalizable for web browsing to various pages. This allows us to give an expected MOS for downloading pictures while a background application is influencing the probability of a device already being in DCH state or still having to be promoted to $\mathrm{DCH}$ state.

Using this methodology, we study the influence of background traffic on the QoE for two background applications with different traffic characteristics. In Figure 8(a), we assume that the user is running the Twitter application as a background process. The application is set to update the users' status feed every 5 minutes. In Figure 8(b), the user is running the Skype application as a background application. This application sends keep-alive messages every 20 seconds. For each application, we assume that the three-state network model with $T_{\mathrm{DCH}}$ settings of $1,4,8$, and 16 seconds is used. We always set $T_{\mathrm{FACH}}=2 \cdot T_{\mathrm{DCH}}$. In both figures, we show the 


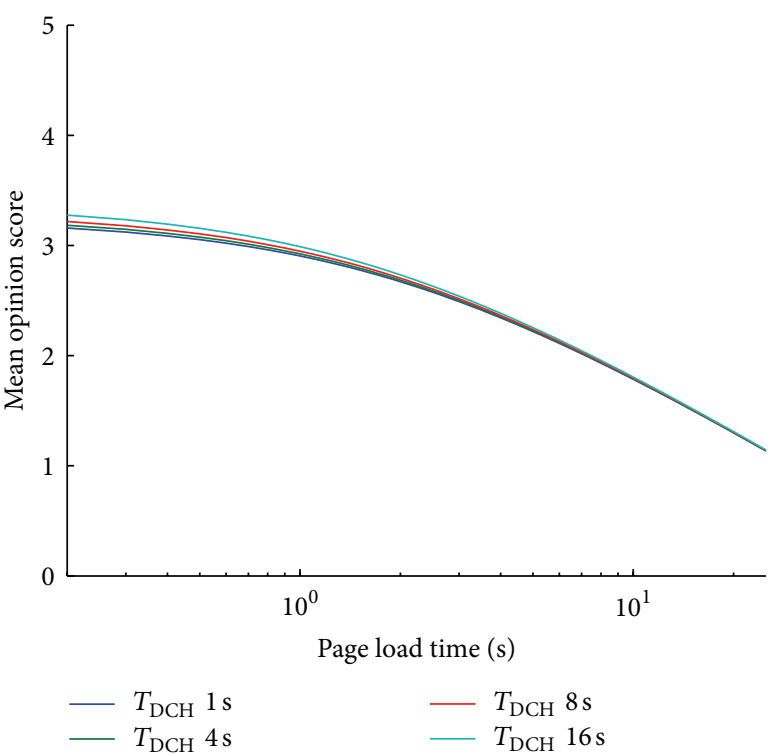

(a) Background traffic generated by Twitter

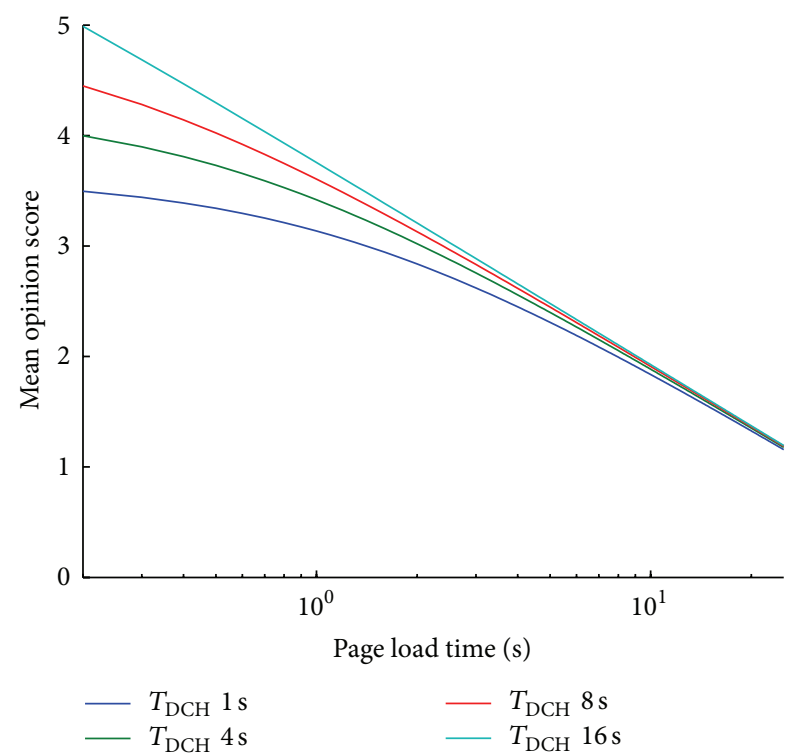

(b) Background traffic generated by Skype

FIgURE 8: Perceived QoE for loading a page with existing background traffic.

assumed page load time, as provided by the network, on the $x$-axis for values from 0.2 seconds to 25 seconds. We assume that 0.1 seconds is a lower bound because page load times lower than 0.1 seconds are not distinguishable [21] by humans. The calculated MOS values are given on the $y$-axis.

The picture downloads with the background traffic generated by the Twitter application result in MOS values beginning at 3.15 for $T_{\mathrm{DCH}}=1$ second, 3.18 for $T_{\mathrm{DCH}}=4$ seconds, 3.21 for $T_{\mathrm{DCH}}=8$ seconds, and 3.27 for $T_{\mathrm{DCH}}=16$ seconds, respectively. With increasing page load time, the MOS again decreases. This behaviour is due to the fact that the Twitter application periodically sends traffic every 5 minutes. Then, no further activity occurs until the next refresh occurs. In this time, the UE transits to Idle state. This traffic characteristic causes an high probability of a user encountering the device in an Idle state. In contrast, downloading pictures with the Skype application generating background traffic causes different MOS values. For a page load time of 0.2 seconds, the MOS value with $T_{\mathrm{DCH}}=1$ second is 3.49 , with $T_{\mathrm{DCH}}=$ 4 seconds is 3.99 , with $T_{\mathrm{DCH}}=8$ seconds is 4.44 , and finally with $T_{\mathrm{DCH}}=16$ seconds is 4.99 , respectively. For increasing page load times, the MOS decreases. This increased MOS values occur because of the high frequency of traffic sent by the Skype application. Here, every 20 seconds, traffic is sent. This means that even for relatively low values of $T_{\mathrm{DCH}}$, the user has a high probability of encountering a state where no promotion delay is required before the actual page load time can begin.

From these studies, we can conclude that, when considering QoE on mobile devices, not only the page load time caused by the network but also additional delays caused by the state of the device should be considered. As shown on two examples, this state can be affected by other applications which are running in the background and generate traffic.

\section{Conclusion}

In this study, we investigate the influence of both application traffic characteristics and network configuration on the signalling load at the RNC as well as the QoE for the user. We consider two different influence factors on QoE: power consumption and page load times for web browsing.

First, we consider the power consumption at the UE. The network operator can reduce the signalling load at the RNC by increasing a network parameter, the $T_{\mathrm{DCH}}$ timer, which determines the time a UE remains connected to the network. However, increasing this timer also increases the battery drain at the UE. While it is possible to find a tradeoff between signalling load and battery drain for any single application, we show that for a set of applications, this is not possible. A network parameter, which might be optimal for one application, may cause another application to generate either a high signalling load or an increased battery drain. Additionally, we consider the influence of background applications on the web QoE. For mobile applications, the page load time may be increased if the UE is currently disconnected and has to connect to the network before obtaining the requested content. If another application is already running, the device may, depending on the traffic pattern of the application, already be online, that is, connected to the radio access network. We suggest to consider this when performing QoE studies for mobile users.

When considering ways to improve mobile networks, many different players, metrics, and tradeoffs exist. We highlighted one examples of such a tradeoff, that is, signalling frequency versus power consumption, and discussed the influence of the current optimisation parameters and the network timers on another. However, many additional tradeoffs exist. For example, the mobile operator has to balance the use of radio resources with the number of 
generated signalling frequencies. Furthermore, application providers seek to improve the user experience which usually results in a higher frequency of network polls, creating additional signalling traffic. The high number of tradeoffs and involved actors in this optimisation problem indicates that the current optimisation technique used by operators is no longer sufficient.

Approaches like Economic Traffic Management or Design for Tussle could be applied to find an acceptable tradeoff for all parties. In economic traffic management all, participating entities share information in order to enable collaboration. This collaboration allows for a joint optimisation of the tradeoff. Design for tussle aims to resolve tussles at run time, instead of design time. This prevents the case that one actor has full control over the optimisation problem, which would likely result in the actor choosing a tradeoff only in its favour, ignoring all other participants.

One example of an actor providing information for another in order to optimise the total system would be UE vendor could provide interfaces for application developers to use when sending data. These interfaces would schedule data to be transmitted in such a way that signalling load and battery drain would be reduced, if the application's requirements allow for it. Until such interfaces exist, application developers could consider the effect of the traffic their applications produce both on the UE and the network. To this end, we will provide a web application as future work, which will allow application developers to upload and analyse their applications' traffic according to different network models.

\section{Acknowledgments}

This work was partly funded by the Deutsche Forschungsgemeinschaft (DFG) under Grants HO 4770/1-1 and TR257/311. The authors would like to thank Florian Wamser for the fruitful discussions. The authors alone are responsible for the content.

\section{References}

[1] F. Cuadrado and J. Dueñas, "Mobile application stores: success factors, existing approaches, and future developments," IEEE Communications Magazine, vol. 50, no. 11, pp. 160-167, 2012.

[2] Cisco, "Cisco visual networking index: global mobile data traffic forecast update, 2011-2016," White Paper, 2012.

[3] TS 25.331, "Radio Resource Control (RRC); Protocol specification," 3GPP Std., 2012.

[4] F. Qian, Z. Wang, A. Gerber, Z. Mao, S. Sen, and O. Spatscheck, "Profiling resource usage for mobile applications: a cross-layer approach," in Proceedings of the 9th International Conference on Mobile Systems, Applications, and Services (MobiSys '11), pp. 321334, July 2011.

[5] F. Qian, Z. Wang, A. Gerber, Z. M. Mao, S. Sen, and O. Spatscheck, "Characterizing radio resource allocation for 3G networks," in Proceedings of the 10th Internet Measurement Conference (IMC '10), pp. 137-150, Melbourne, Australia, November 2010.

[6] Nokia Siemens Networks, "Understanding smartphone behavior in the network," White Paper, 2011.
[7] C. Yang, "Huawei communicate: weather the signalling storm," White Paper, 2011.

[8] S. Ickin, K. Wac, M. Fiedler, L. Janowski, J. Hong, and A. Dey, "Factors influencing quality of experience of commonly used mobile applications," IEEE Communications, vol. 50, no. 4, pp. 48-56, 2012.

[9] T. Hoßfeld, D. Hausheer, F. Hecht et al., "An economic traffic management approach to enable the TripleWin for users, ISPs, and overlay providers," in Towards the Future Internet-A European Research Perspective, G. Tselentis, J. Domingue, A. Galis et al., Eds., pp. 24-34, IOS Press Books Online, May 2009.

[10] D. D. Clark, J. Wroclawski, K. R. Sollins, and R. Braden, "Tussle in cyberspace: defining tomorrow's internet," IEEE/ACM Transactions on Networking, vol. 13, no. 3, pp. 462-475, 2005.

[11] Trilogy Project, "D2-lessons in "designing for tussle" from case studies," Deliverable of the Trilogy Project ICT-216372, 2008.

[12] S. Egger, P. Reichl, T. Hoßfeld, and R. Schatz, "Time is bandwidth? Narrowing the gap between subjective time perception and quality of experience," in Proceedings of the International Conference on Communications (ICC '12), p. 1325, Ottawa, Canada, June 2012.

[13] P. H. J. Perälä, A. Barbuzzi, G. Boggia, and K. Pentikousis, "Theory and practice of RRC state transitions in UMTS networks," in Proceedings of the Global Communication Conference (GLOBECOM '09), pp. 1-6, Honolulu, Hawaii, USA, December 2009.

[14] GSMAssociation and others, "Network efficiency task force fast dormancy best practices," White Paper, May 2010.

[15] F. Qian, Z. Wang, A. Gerber, Z. M. Mao, S. Sen, and O. Spatscheck, "TOP: tail optimization protocol for cellular radio resource allocation," in Proceedings of the 18th IEEE International Conference on Network Protocols (ICNP '10), pp. 285-294, Kyoto, Japan, October 2010.

[16] TR 22.801, "Study on non-MTC Mobile Data Applications impacts (Release 11)," 3GPP Std., 2011.

[17] A. Díaz Zayas and P. Merino Gómez, "A testbed for energy profile characterization of IP services in smartphones over live networks," Mobile Networks and Applications, vol. 15, no. 3, pp. 330-343, 2010

[18] C. Schwartz, T. Hoßfeld, F. Lehrieder, and P. Tran-Gia, "Performance analysis of the trade-off between signalling load and power consumption for popular smartphone apps in $3 \mathrm{G}$ networks," Tech. Rep. 485, University of Würzburg, January 2013.

[19] M. Calder and M. K. Marina, "Batch scheduling of recurrent applications for energy savings on mobile phones," in Proceedings of the 7th Annual IEEE Communications Society Conference on Sensor, Mesh and Ad Hoc Communications and Networks (SECON '10), pp. 1-3, Boston, Mass, USA, June 2010.

[20] E. Vergara and S. Nadjm-Tehrani, “"Energy-aware cross-layer burst buffering for wireless communication," in Proceedings of the Conference on Future Energy Systems: Where Energy, Computing and Communication Meet, p. 24, Madrid, Spain, May 2012.

[21] S. Egger, T. Hoßfeld, R. Schatz, and M. Fiedler, "Waiting times in quality of experience for web based services," in Proceedings of the Workshop on Quality of Multimedia Experience (QoMEX '12), pp. 86-96, Yarra Valley, Australia, July 2012. 

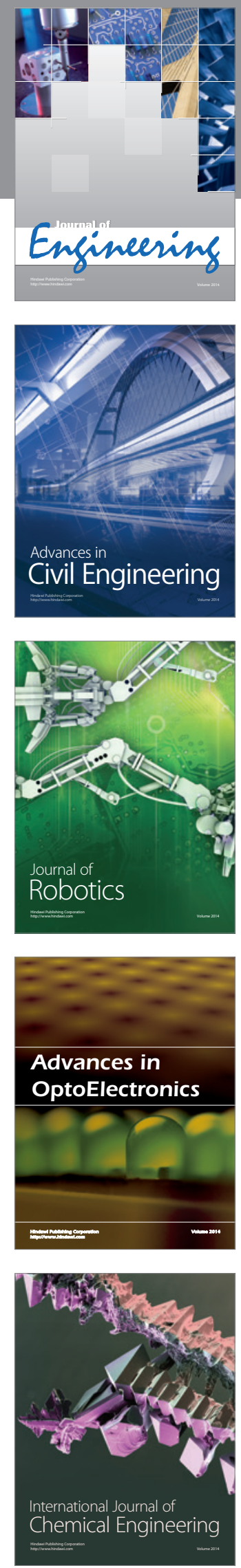

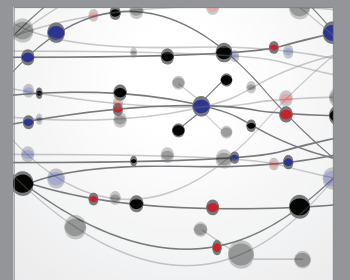

The Scientific World Journal
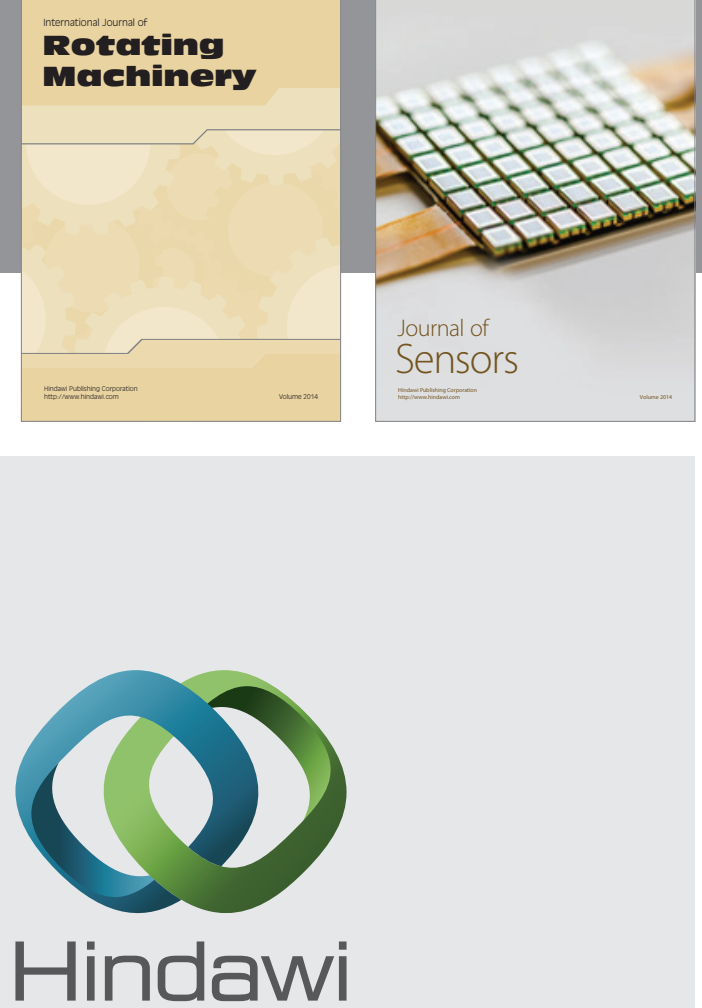

Submit your manuscripts at http://www.hindawi.com
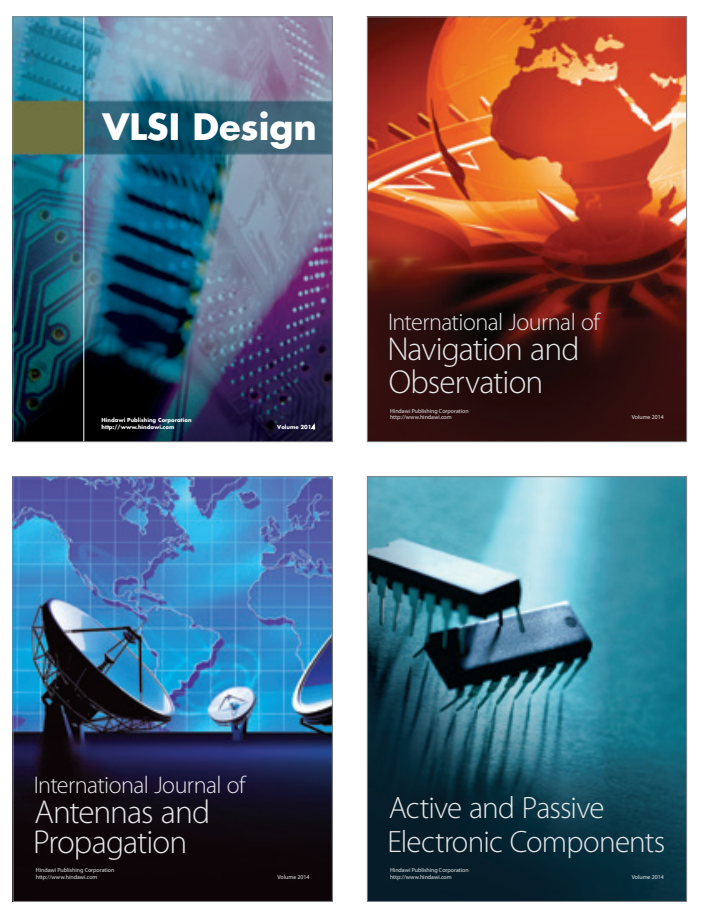
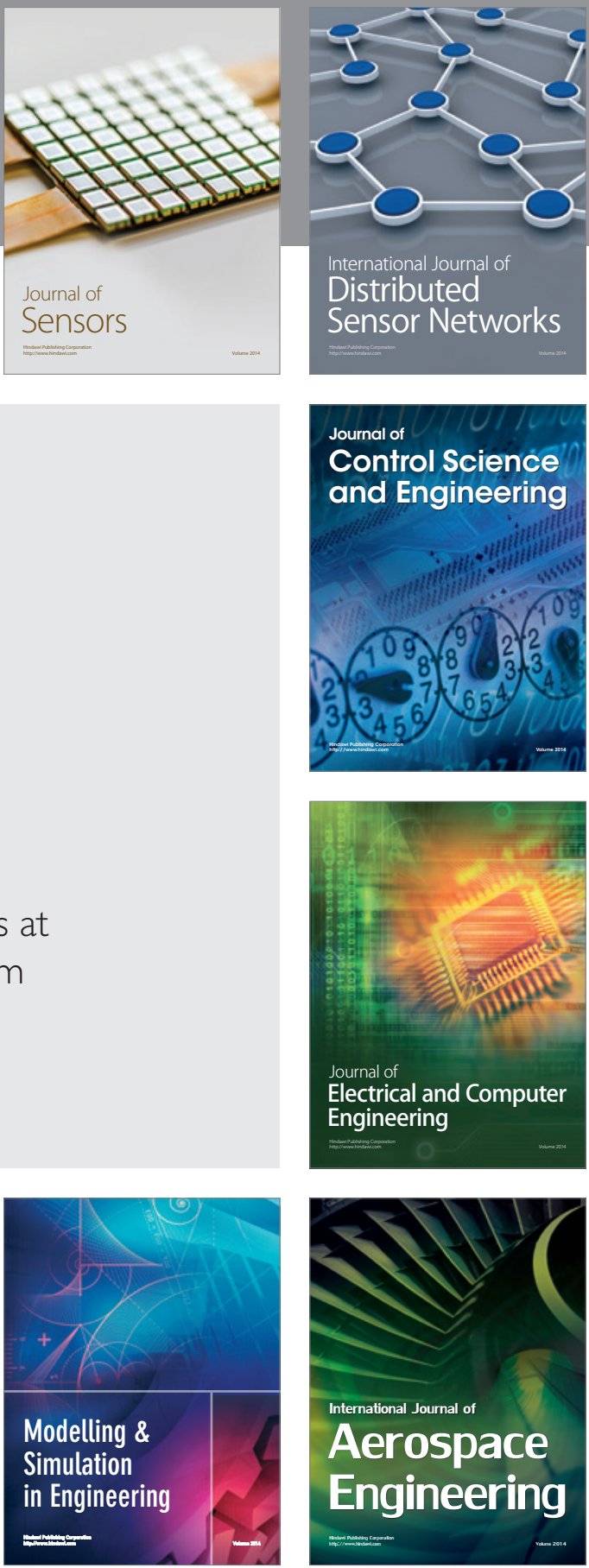

Journal of

Control Science

and Engineering
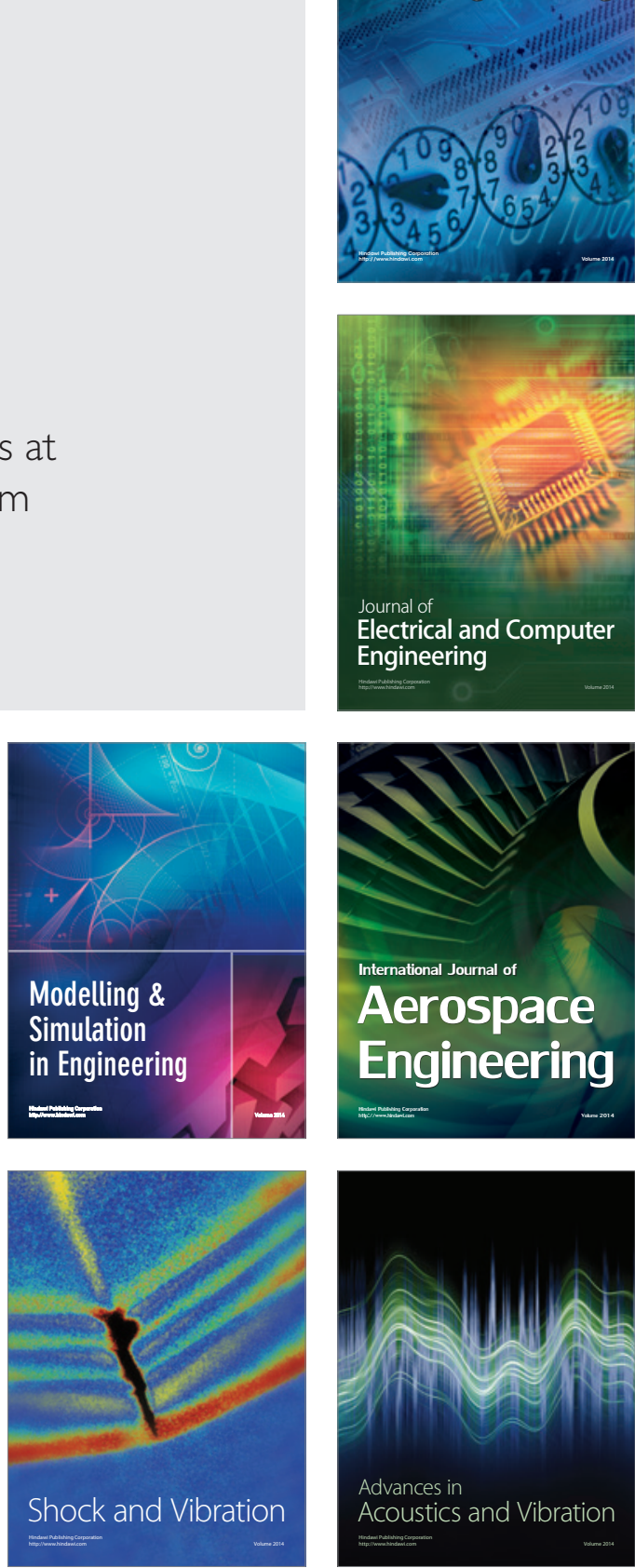